\title{
Classification of Student's Confusion Level in E-Learning using Machine Learning
}

\author{
Bikram Kumar, Deepak Gupta, Rajat Subhra Goswami
}

\begin{abstract}
With the advancement of technology, the traditional mode of teaching-learning pedagogy has evolved into online education system as it is easily accessible. But, it is very difficult to detect whether the students are 'confused' or 'not confused' while watching online videos. Getting confused while watching online videos is one of the root causes of less performance of the students. Keeping in mind the above statements, we would like to investigate whether the students are 'confused' or 'not confused' while watching Massive Open Online Course (MOOC) videos. There are a lot of studies that prove electroencephalogram (EEG) signals behave differently as we are in different conditions such as happy, sad, angry, etc. So, in this paper, we have applied several supervised learning algorithms to detect if the students are 'confused' or 'not confused' while watching MOOC videos using EEG data. The results of this paper show that machine learning is a potential technique, for the analysis of EEG data that can detect the confusion level of the students which is comparable to human observation for predicting the confusion level of the students that can improve the quality of online education system.
\end{abstract}

Keywords: Confusion, EEG, Machine Learning, MOOC, Supervised Learning

\section{INTRODUCTION}

$\mathbf{U}$ nlike classroom education, MOOC is a non-campus, large scale, online education platform where any number of students can access any number of online courses via the Internet and this is increasing day-by-day [1]. But, it comes with its own pitfalls. Thompson showed the behavior of the students who were not fully attentive toward the correspondence-based distance education program [2]. There is a huge difference between classroom education and online education. In a classroom education, the educator can judge whether the students could understand the topics or not by verbal questioning, body language, etc and make them understand where this is a serious issue in online education. Sharma suggests some guidelines for the stakeholders in e-learning for better online course design [3]. Sublett showed the known fact about online courses and the impacts of the online courses on the community college students with respect to course completion, persistence, transfer and degree completion [4]. Now, these days MOOC providers provide interactive sessions where the instructor and the students

Revised Manuscript Received on December 15, 2019.

* Correspondence Author

Bikram Kumar, M.Tech scholar of CSE Dept, NIT Arunachal Pradesh, Naharlagun, India. Email: biikramkumar@gmail.com

Deepak Gupta*, Assistant Professor of CSE Dept., NIT Arunachal Pradesh, Naharlagun, India. Email: deepak@nitap.ac.in

Rajat Subhra Goswami, Assistant Professor of CSE Dept., NIT Arunachal Pradesh, Naharlagun, India. Email: rajat@nitap.ac.in can discuss several things and also provide feedback form to the students to get the student's review. No one can deny that online education provides ample amount of benefits to the students. But, still, the lack of a classroom is a big concern. But, detection of the students confusion level while watching MOOC videos is a key idea to make online education system more efficient.

EEG is typically a non-invasive medical diagnostic technique, where electrodes are put on the scalp of the subject to record the electrical activity of the brain [5]. The variation of the voltage between the neuron of the brain is measured by the EEG signal. EEG estimates both frequency and amplitude of electrical activity produced from the brain [6]. EEG can give millisecond range temporal resolution which is not possible with any high-resolution imaging technique [7]. Because of this, many researchers use EEG data to solve brain-related problems. H. Berger, the German physiologist recored the EEG of a human for the first time in 1924 [8]. EEG data is normally used to diagnose epilepsy. It can likewise be utilized to recognize sleep disorders, coma, encephalopathy, brain death, emotions like anger, disgust, happy, sad, fear and many more. EEG signal varies from situation-to-situation. So, we assumed that the EEG signal of a student who is 'confused' will differ from a student who is 'not confused'. So, in this paper, we use EEG data to determine the student's confusion level while watching MOOC videos.

\section{RELATED WORK}

There are many researchers who are using different Machine Learning algorithms along with EEG data to perform different tasks like detection of emotion, Alzheimer, drowsiness of car driver, etc. Yeo et al. used the support vector machine (SVM) for detecting car driver's drowsiness during car driving using EEG data [9]. Wang et al. gathered EEG from 10 college students to check the confusion level of the students while watching online videos using Gaussian Naive Bayes classifier [10]. Mampusti et al. used EEG data for measuring academic affective states of the students using three supervised learning algorithms namely K-Nearest Neighbors, SVM and Multilayer Perceptron (MLP) respectively [11]. Subasi and Gursoy used EEG data along with PCA, ICA and LDA to extract features and SVM to predict epileptic seizure or not [12]. Ni et al. used EEG data and bidirectional LSTM recurrent neural network to check whether a student is 'confused' or 'not confused' while watching online course videos [13]. Edla et al. 
lead an investigation to gather EEG data from 40 human brains and used techniques to extract features with random forest classifier for the classification of mental state of human into two classes those were concentration and meditation [14]. For emotion detection, Mangalagowri and Raj used EEG data and extract features using 'db4' wavelet using multilevel decomposition and used Feed Forward Backpropogation algorithm [15]. Hajinoroozi et al. used Deep Belief Networks (DBN) to extract features and dimensionality reduction from EEG data to predict the cognitive states of the driver [16]. The results showed that $\mathrm{DBN}-\mathrm{C}$ is a potential technique to extract features. Petrosian et al. showed that using long-term EEG signals recurrent neural networks can recognize Alzheimer disease symptoms [17]. Abdulla et al. performed EEG signal analysis with extreme machine learning algorithm to develop a technique to detect the sleep stages based on EEG signals [18]. Hussain et al. used five different supervised learning algorithms and performed k-fold cross-validation to detect the student difficulties using session data [19]. Lin and Kao used machine learning algorithms to perform mental effort detection in e-learning using EEG data [20]. Bacos explored human-centered techniques of using technology in education [21]. Raheel, Majid, and Anwar used EEG data and from the data thirteen different features were extracted to classify five different expressions namely looking up, looking down, smile, eye-wink and eye blink using four different classifiers namely K-nearest neighbor, naïve bayes, SVM and MLP [22]. Zhou et al. used EEG data and proposed an end-to-end method for the analysis of EEG data to detect the confusion state of student in learning [23].

\section{PROBLEM STATEMENT}

Online education has become a new means in the field of education as multiple number of students can access a huge number of courses through the internet. It is emerging day-by-day as students can learn new things, gain knowledge and skill from different sources. But, there is a drawback of online education. There is no absolute way for the instructor to know whether the participant students are fully 'attentive' or 'confused'. Because, if the students are 'confused' while watching the videos they would not be able to gain knowledge. But, if the instructor would be able to know that the student is 'confused', he would be able to make the student understand the topic and there would be many more chances for making the online education platform more effectively efficient.

To detect the confusion level of the students while watching the MOOC videos, here we have used the EEG data. Since the confusion levels (pre-defined and user-defined) are either true or false, this is a binary class classification problem. So, we have applied several supervised learning algorithms like Decision Tree, Random Forest, Boosting algorithms, Bagging algorithms, SVM with different kernels, etc. to detect both pre-defined confusion level and user-defined confusion level. Figure 1 represents the confusion detection framework.

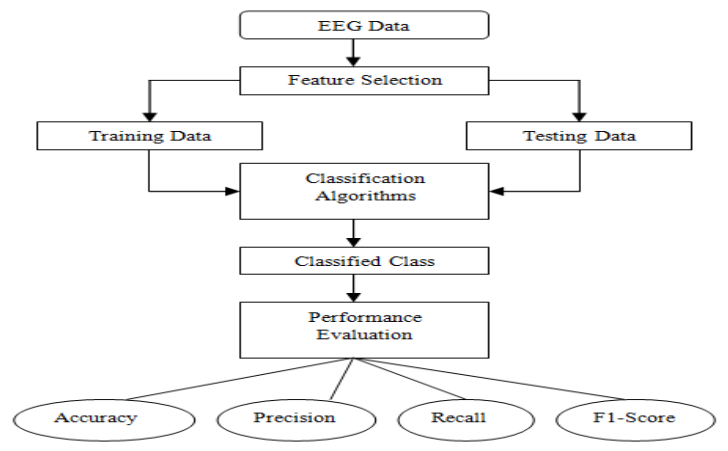

Fig. 1. Student's Confusion detection framework

\section{DATASET DESCRIPTION}

The EEG data were gathered from 10 college students while they were watching MOOC videos. 20 videos were prepared, 10 of each category i.e. 10 pre-labeled as "easy", where 10 were pre-labeled as "difficult". Duration of each video was 2 minutes. The dataset "Confused student EEG brainwave data" is available at Kaggle [24].

Students were put on Mindset EEG devices. These devices were single-channel wireless devices and are used to measure the functioning of the frontal lobe. The devices operate by measuring the voltage between an electrode fixed on the forehead and two electrodes each of which are connected with an ear. Students gave their rating about their confusion level on a 1-7 scale, where 1 corresponds to least confusing and 7 corresponds to most confusing. These levels were quantified into two classes of which whether a student is 'confused' or 'not confused'. In the dataset 0 represents 'not confused' students and 1 represents 'confused' students. There are two labels: the pre-defined level has been assigned as per the experiment design and user-defined level has been assigned as per the students' review.

\section{CLASSIFICATION FEATURES}

For each student watching the videos, features were extracted at the sampling frequency of $2 \mathrm{~Hz}$. There is total of 13 features in the dataset out of which 2 features are "Subject id" and "Video id" that range from 0 to 9 and we don't use them for classification. "Attention" measures the focus of the students and "Meditation" measures the calmness and "Raw" is the average of the original EEG signal. Table I shows the features that we have considered to classify class labels for 'confused' or 'not confused' students classification.

Table I: Feature's description of the dataset

\begin{tabular}{|c|c|c|}
\hline Feature & Sampling Rate & Statistic \\
\hline Attention & $1 \mathrm{~Hz}$ & Mean \\
\hline Meditation & $1 \mathrm{~Hz}$ & Mean \\
\hline Raw & $512 \mathrm{~Hz}$ & Mean \\
\hline Delta & $8 \mathrm{~Hz}$ & Mean \\
\hline Theta & $8 \mathrm{~Hz}$ & Mean \\
\hline Alpha1 & $8 \mathrm{~Hz}$ & Mean \\
\hline Alpha2 & $8 \mathrm{~Hz}$ & Mean \\
\hline Beta1 & $8 \mathrm{~Hz}$ & Mean \\
\hline Beta2 & $8 \mathrm{~Hz}$ & Mean \\
\hline \multicolumn{2}{|c|}{} \\
$\begin{array}{l}\text { Published By: } \\
\text { Blue Eyes Intelligence Engineering } \\
\text { \& Sciences Publication }\end{array}$
\end{tabular}




\begin{tabular}{|l|l|l|}
\hline Gamma1 & $8 \mathrm{~Hz}$ & Mean \\
\hline Gamma2 & $8 \mathrm{~Hz}$ & Mean \\
\hline
\end{tabular}

\section{EXPERIMENTAL SETUP}

To identify the class label of the students whether the students are 'confused' or 'not confused' while watching MOOC videos, we have evaluated 32 classifiers of different category. The experimental environment is an Intel (R) Core (TM) i5-4590 CPU $3.30 \mathrm{GHz}, 4$ GB RAM, a desktop running Windows 8 (64-bit), Python 3.7.3, Pandas 0.25.1, NumPy 1.17.3, Scipy 1.3.1, Scikit-learn 0.21.3, Matplotlib 3.1.1.

Here, we have evaluated 32 supervised machine learning algorithms to predict if the students are 'confused' or 'not confused' while watching MOOC videos. As state before, we have two different levels i.e. one is pre-defined level which has been assigned according to the experiment design and another is user-defined level which has been assigned as per the students review, we have applied 32 classifiers to predict both the confusion level.

Classification of pre-defined level: We take $70 \%$ of the dataset to train the classifiers and the remaining $30 \%$ to test the classifiers. So, it has 3844 test cases out of which 2016 are 'not confused' students and 1828 'confused' students. 5-fold cross-validation is performed to find the optimum parameter of each classifier. For each 5-fold cross-validation, we randomly divided the entire dataset into 5 folds, out of which 4 folds are used to train the classifier and remaining 1 fold is used to evaluate the classifier's performance.

Classification of user-defined level: We take $70 \%$ of the dataset to train the classifiers and the remaining $30 \%$ to test the classifiers. So, it has 3844 test cases out of which 1882 are 'not confused' students and 1962 'confused' students. Similarly, we perform 5-fold cross-validation to find out the optimum parameters for each classifier.

Table II: Performance of classifiers for detection of pre-defined confusion level

\begin{tabular}{|c|c|c|c|c|c|}
\hline Classifier & Parameter & Accuracy & Precision & Recall & F1-Score \\
\hline KnearestNeighbor & n_neighbors $=1$ & 54.86 & 0.55 & 0.55 & 0.55 \\
\hline Logistic Regressoion & $\mathrm{C}=1 \mathrm{e}-02$ & 53.75 & 0.53 & 0.54 & 0.51 \\
\hline Logistic RegressoionCV & solver='liblinear', penalty='l1' & 53.88 & 0.54 & 0.54 & 0.52 \\
\hline Quadratic Discriminant Analysis & reg_param=1, store_covarience='True' & 53.38 & 0.53 & 0.53 & 0.53 \\
\hline Ridge Classifier & alpha $=1 \mathrm{e}-05$ & 53.38 & 0.53 & 0.53 & 0.5 \\
\hline Ridge ClassifierCV & alphas $=[1 \mathrm{e}-04,1 \mathrm{e}-03,1 \mathrm{e}-02,1 \mathrm{e}-01,1,1 \mathrm{e}+01,1 \mathrm{e}+02,1 \mathrm{e}+03]$ & 53.38 & 0.53 & 0.53 & 0.5 \\
\hline Decision Tree & splitter='random',criterion='entropy' & 56.32 & 0.56 & 0.56 & 0.56 \\
\hline Gaussian Naive Bayes & var_smoothing $=10$ & 52.44 & 0.28 & 0.52 & 0.36 \\
\hline Perceptron & alpha $=1 \mathrm{e}-05, \mathrm{tol}=1 \mathrm{e}-05$ & 49.11 & 0.62 & 0.49 & 0.35 \\
\hline Linear SVM & $\mathrm{C}=1 \mathrm{e}-03$ & 52.52 & 0.75 & 0.52 & 0.36 \\
\hline SVM (Kernel='linear') & $\mathrm{C}=1 \mathrm{e}-05$,gamma $=2^{\wedge}-5$ & 52.11 & 0.52 & 0.52 & 0.52 \\
\hline SVM (kernel='rbf') & $\mathrm{C}=1$, gamma $=2^{\wedge}-5$ & 55.18 & 0.76 & 0.55 & 0.42 \\
\hline SVM (Kernel='sigmoid') & $\mathrm{C}=1 \mathrm{e}-05$, gamma $=2^{\wedge}-5$ & 52.44 & 0.28 & 0.52 & 0.36 \\
\hline Passive Aggressive Classifier & $\mathrm{C}=1 \mathrm{e}-05$,validation_fraction $=0.1$ & 50.62 & 0.5 & 0.51 & 0.51 \\
\hline Gaussian Process & Default & 55.18 & 0.76 & 0.55 & 0.42 \\
\hline SGD Classifier & Alpha $=10$, penalty='11' & 52.18 & 0.52 & 0.52 & 0.52 \\
\hline Multi Layer Perceptron & Alpha $=10^{\wedge} 5$,hidden_layer_sizes $=9$ & 52.47 & 0.52 & 0.52 & 0.47 \\
\hline Random Forest & n_estimators $=64$ & 61.29 & 0.61 & 0.61 & 0.61 \\
\hline Extra Tress & criterion='gini',max_depth=19,n_estimators $=80$ & 60.4 & 0.6 & 0.6 & 0.6 \\
\hline Ada Boosting & learning_rate $=1, \mathrm{n}$ _estimators $=58$ & 54.85 & 0.55 & 0.55 & 0.55 \\
\hline Gradient Boosting & $\begin{array}{c}\text { learning_rate }=0.05, \text { max_depth }=24, \text { n_estimators }=200, \text { sub } \\
\text { sample }=0.1\end{array}$ & 59.21 & 0.59 & 0.59 & 0.59 \\
\hline XG Boosting & $\begin{array}{c}\text { colsample_bytree }=0.7, \text { gamma }=0.1 \text {,learning_rate }=0.05, \mathrm{~m} \\
\text { ax_depth }=15, \mathrm{~min} \_ \text {child_weight }=5\end{array}$ & 60.33 & 0.6 & 0.6 & 0.6 \\
\hline Bagging with Decision Tree & n_estimators $=250$ & 61.78 & 0.62 & 0.62 & 0.62 \\
\hline Bagging with Random Forest & n_estimators $=55$ & 61.89 & 0.62 & 0.62 & 0.62 \\
\hline Bagging with Extra Trees & n_estimators $=50$ & 61 & 0.61 & 0.61 & 0.61 \\
\hline Bagging with Logistic Regression & n_estimators $=15$ & 53.46 & 0.53 & 0.53 & 0.52 \\
\hline Bagging with SVM(kernel='linear') & n_estimators $=10$ & 48.23 & 0.49 & 0.48 & 0.46 \\
\hline Bagging with SVM(kernel='rbf') & n_estimators $=30$ & 54.84 & 0.76 & 0.55 & 0.41 \\
\hline $\begin{array}{c}\text { Bagging with } \\
\text { SVM(kernel='sigmoid })\end{array}$ & n_estmators $=1$ & 52.44 & 0.28 & 0.52 & 0.36 \\
\hline
\end{tabular}

Table III: Performance of classifiers for detection of user-defined confusion level

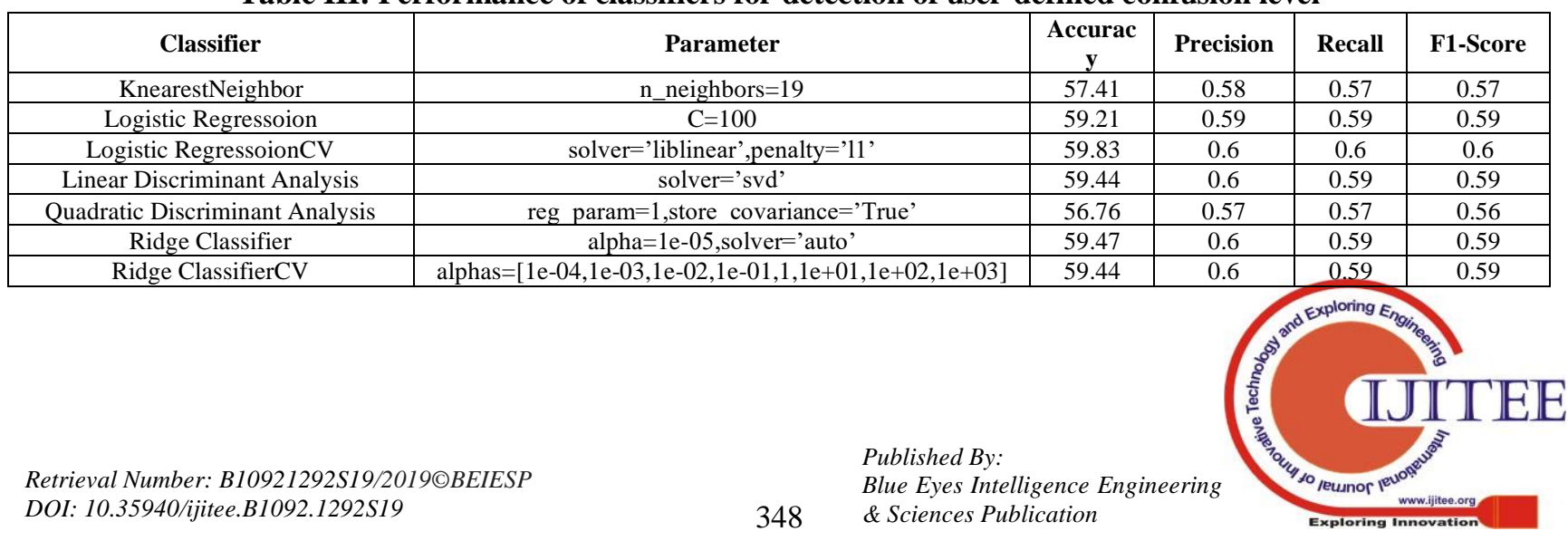




\begin{tabular}{|c|c|c|c|c|c|}
\hline Decision Tree & splitter='random',criterion='entropy' & 59 & 0.59 & 0.59 & 0.59 \\
\hline Gaussian Naive Bayes & var_smoothing=1 & 55.85 & 0.57 & 0.56 & 0.54 \\
\hline Perceptron & alpha $=1 \mathrm{e}-05$, tol $=1 \mathrm{e}-05$ & 53.77 & 0.56 & 0.54 & 0.48 \\
\hline Linear SVM & $\mathrm{C}=0.0001$ & 53.02 & 0.54 & 0.53 & 0.52 \\
\hline SVM (Kernel='linear') & $\mathrm{C}=1 \mathrm{e}-05$, gamma $=2^{\wedge}-5$ & 52.11 & 0.52 & 0.52 & 0.52 \\
\hline SVM (kernel='rbf') & $\mathrm{C}=1 \mathrm{e}-05$, gamma $=2^{\wedge}-5$ & 51.04 & 0.26 & 0.51 & 0.34 \\
\hline SVM (Kernel='sigmoid') & $\mathrm{C}=1 \mathrm{e}-05$,gamma $=2^{\wedge}-5$ & 51.04 & 0.26 & 0.51 & 0.34 \\
\hline Passive Aggressive Classifier & $\mathrm{C}=1 \mathrm{e}-05$,validation_fraction $=0.1$ & 53.59 & 0.54 & 0.54 & 0.52 \\
\hline Gaussian Process & Default & 53.12 & 0.76 & 0.53 & 0.41 \\
\hline NuSVC & Gamma $=2^{\wedge}-5, \mathrm{nu}=0.1$ & 51.04 & 0.26 & 0.51 & 0.34 \\
\hline SGD Classifier & Alpha $=0.1$, penalty='11' & 51.24 & 0.51 & 0.51 & 0.51 \\
\hline Multi Layer Perceptron & Alpha $=10^{\wedge} 5$,hideen_layer_sizes $=8$ & 58.38 & 0.59 & 0.58 & 0.58 \\
\hline Random Forest & n_estimators $=93$ & 65.89 & 0.66 & 0.66 & 0.66 \\
\hline Extra Tress & max_depth=28, n_estimators $=77$ & 64.2 & 0.64 & 0.64 & 0.64 \\
\hline Ada Boosting & learning_rate $=1, \mathrm{n}$ _estimators $=45$ & 61.13 & 0.61 & 0.61 & 0.61 \\
\hline Gradient Boosting & $\begin{array}{c}\text { learning_rate }=0.01, \text { max_depth }=29, \text { n_estimators }=200, \text { sub } \\
\text { sample }=0.1\end{array}$ & 64.07 & 0.64 & 0.64 & 0.64 \\
\hline XG Boosting & $\begin{array}{c}\text { colsample_bytree }=0.7 \text {,gamma }=0.3 \text {, learning_rate }=0.05, \mathrm{~m} \\
\qquad \mathrm{ax} \_ \text {depth }=12\end{array}$ & 65.01 & 0.65 & 0.65 & 0.65 \\
\hline Bagging with Decision Tree & n_estimators $=120$ & 61.63 & 0.62 & 0.62 & 0.62 \\
\hline Bagging with Random Forest & n_estimators $=20$ & 66.6 & 0.67 & 0.67 & 0.67 \\
\hline Bagging with Extra Trees & n_estimators $=100$ & 65.69 & 0.66 & 0.66 & 0.66 \\
\hline Bagging with Logistic Regression & n_estimators $=15$ & 59.2 & 0.59 & 0.59 & 0.59 \\
\hline Bagging with SVM(kernel='linear') & n_estimators $=50$ & 48.93 & 0.63 & 0.49 & 0.35 \\
\hline Bagging with SVM(kernel='rbf') & n_estimators $=20$ & 54.63 & 0.76 & 0.55 & 0.41 \\
\hline $\begin{array}{c}\text { Bagging with } \\
\text { SVM(kernel='sigmoid })\end{array}$ & n_estimators $=100$ & 52.44 & 0.28 & 0.52 & 0.36 \\
\hline Voting Classifier & $\begin{array}{c}\text { estimators }=[\text { LogisticRegression, RandomForest, } \\
\text { GaussianNB] }\end{array}$ & 64.41 & 0.65 & 0.64 & 0.64 \\
\hline
\end{tabular}

Table IV: Classifier's rank to detect user-defined level

\begin{tabular}{|c|c|c|}
\hline \multirow{2}{*}{ Classifier } & \multicolumn{2}{|c|}{ Rank } \\
\hline & Accuracy & F1-score \\
\hline KnearestNeighbor & 18 & 18 \\
\hline Logistic Regression & 14 & 13.5 \\
\hline Logistic RegressionCV & 10 & 10 \\
\hline Linear Discriminant Analysis & 12.5 & 13.5 \\
\hline Quadratic Discriminant Analysis & 19 & 19 \\
\hline Ridge Classifier & 11 & 13.5 \\
\hline Ridge ClassifierCV & 12.5 & 13.5 \\
\hline Decision Tree & 16 & 13.5 \\
\hline Gaussian Naïve Bayes & 20 & 20 \\
\hline Perceptron & 22 & 25 \\
\hline Linear SVM & 25 & 22 \\
\hline SVM(kernel='linear') & 27 & 22 \\
\hline SVM(kernel='rbf') & 30 & 31 \\
\hline SVM(kernel='sigmoid') & 30 & 31 \\
\hline Passive Aggressive Classifier & 23 & 22 \\
\hline Gaussian Process & 24 & 26.5 \\
\hline NuSVC & 30 & 31 \\
\hline SGD Classifier & 28 & 24 \\
\hline Multi Layer Perceptron & 17 & 17 \\
\hline Random Forest & 2 & 2.5 \\
\hline Extra Tress & 6 & 6 \\
\hline Ada Boosting & 9 & 9 \\
\hline Gradient Boosting & 7 & 6 \\
\hline XG Boosting & 4 & 4 \\
\hline Bagging with Decision Tree & 8 & 8 \\
\hline Bagging with Random Forest & 1 & 1 \\
\hline Bagging with Extra Trees & 3 & 2.5 \\
\hline Bagging with Logistic Regression & 15 & 13.5 \\
\hline Bagging with SVM(kernel='linear') & 32 & 29 \\
\hline Bagging with SVM(kernel='rbf') & 21 & 26.5 \\
\hline Bagging with SVM(kernel='sigmoid') & 26 & 28 \\
\hline Voting Classifier & 5 & 6 \\
\hline
\end{tabular}

Table V: Classifier's rank to detect pre-defined level

\begin{tabular}{|l|c|c|}
\hline \multirow{2}{*}{\multicolumn{2}{|c|}{ Classifier }} & \multicolumn{2}{c|}{ Rank } \\
\cline { 2 - 3 } & Accuracy & F1-score \\
\hline KnearestNeighbor & 13 & 10.5 \\
\hline Logistic Regression & 17 & 17.5 \\
\hline Logistic RegressionCV & 16 & 14.5 \\
\hline Linear Discriminant Analysis & 20.5 & 20 \\
\hline Quadratic Discriminant Analysis & 20.5 & 12 \\
\hline Ridge Classifier & 20.5 & 20 \\
\hline Ridge ClassifierCV & 20.5 & 20 \\
\hline Decision Tree & 9 & 9 \\
\hline Gaussian Naïve Bayes & 26 & 29.5 \\
\hline Perceptron & 31 & 32 \\
\hline Linear SVM & 23 & 29.5 \\
\hline SVM(kernel='linear') & 29 & 14.5 \\
\hline SVM(kernel='rbf') & 11 & 25 \\
\hline SVM(kernel='sigmoid') & 26 & 29.5 \\
\hline Passive Aggressive Classifier & 30 & 17.5 \\
\hline Gaussian Process & 11 & 25 \\
\hline NuSVC & 11 & 25 \\
\hline SGD Classifier & 28 & 14.5 \\
\hline Multi Layer Perceptron & 24 & 22 \\
\hline Random Forest & 3 & 3.5 \\
\hline Extra Tress & 5 & 6 \\
\hline Ada Boosting & 14 & 10.5 \\
\hline Gradient Boosting & 8 & 8 \\
\hline XG Boosting & 6 & 6 \\
\hline Bagging with Decision Tree & 2 & 1.5 \\
\hline Bagging with Random Forest & 1 & 1.5 \\
\hline Bagging with Extra Trees & 4 & 3.5 \\
\hline Bagging with Logistic Regression & 18 & 14.5 \\
\hline Bagging with SVM(kernel='linear') & 32 & 23 \\
\hline Bagging with SVM(kernel='rbf') & 15 & 27 \\
\hline Bagging with SVM(kernel='sigmoid') & 26 & 29.5 \\
\hline Voting Classifier & 7 & 6 \\
\hline
\end{tabular}




\section{PERFORMANCE EVALUATION METRICS}

For classifier's performance evaluation we have used 4 difference matrices: accuracy, precision, recall and F1- score.

A. Accuracy: It is the ratio of correctly labeled points and the total number of points. Accuracy is given by the following:

where,

$$
\text { Accuracy }=(T P+T N) /(T P+T N+F P+F N)
$$

TP: True Positive

TN: True Negative

FP: False Positive

FN: False Negative

B. Precision: It refers to the percentage of results which are relevant. High precision means that an example labeled as positive is positive. It is given by:

$$
\text { Precision }=T P /(T P+F P)
$$

C. Recall: It refers to the total relevant results correctly classified by our algorithm. High recall means class is identified correctly. It is given by:

$$
\text { Recall }=T P /(T P+F N)
$$

D. F1-Score: It gives the weighted mean of precision and recall. It is given by:

\section{F1-Score $=(2 *$ Precision $*$ Recall $) /($ Recall + Precision $)$}

\section{RESULTS AND DISCUSSIONS}

A. User-defined Confusion Level Detection: Table III represents the results of the different classifiers we have used to detect the user-defined confusion level of the students. We found out that for user-defined confusion level detection Bagging with Random Forest, Random Forest and Bagging with Extra Trees classifier gives the best result- their accuracy scores are $66.6 \%, 65.89 \%$ and $65.69 \%$ respectively and their F1-scores are- 0.67, 0.66 and 0.66. On the other hand, Bagging with SVM (kernel='linear'), NuSVC, SVM (kernel='sigmoid') and SVM (kernel='rbf') shows the worst performance, their accuracy scores are- 48.93\%, 51.04\%, $51.04 \%$ and $51.04 \%$ respectively and their F1-scores are$0.35,0.34,0.34$ and 0.34 respectively. Table IV shows the ranks of the different classifiers based on accuracy and F1-score. Table VI represents the confusion matrix for the best two classifiers i.e. Bagging with Random Forest and Random Forest to detect user-defined confusion level. Figure 2 (a) and (b) represents the Receiver Operating Characteristic (ROC) curve for the above two classifiers.

B. Pre-defined Confusion Level Detection: Table II represents the results of the different classifiers we have used to detect the pre-defined confusion level of the students. Among all the 32 classifiers, we found that Bagging with Random Forest, Bagging with Decision Tree and Random Forest shows the best performance - their accuracy scores are $61.89 \%, 61.78 \%$ and $61.29 \%$ respectively, and F1-scores are $0.62,0.62$ and 0.61 respectively.

Table VI: Confusion Matrix to detect user-defined level

\begin{tabular}{|c|c|c|c|c|}
\hline \multirow{2}{*}{ Classifier } & \multicolumn{4}{|c|}{ Confusion Matrix } \\
\cline { 2 - 5 } & $T P$ & $T N$ & $F P$ & $F N$ \\
\hline Bagging with Random Forest & 1226 & 1334 & 628 & 656 \\
\hline Random Forest & 1224 & 1309 & 653 & 658 \\
\hline
\end{tabular}

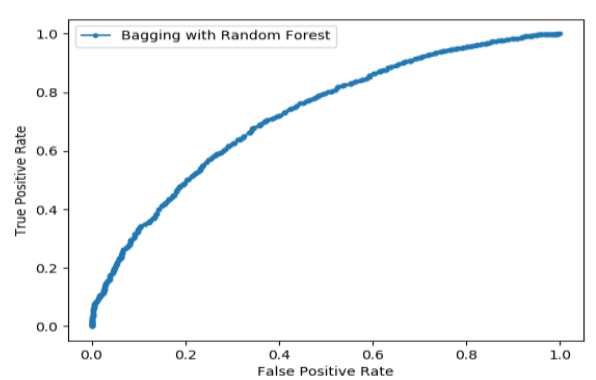

Fig. 2. (a) ROC curve of Bagging with Random Forest

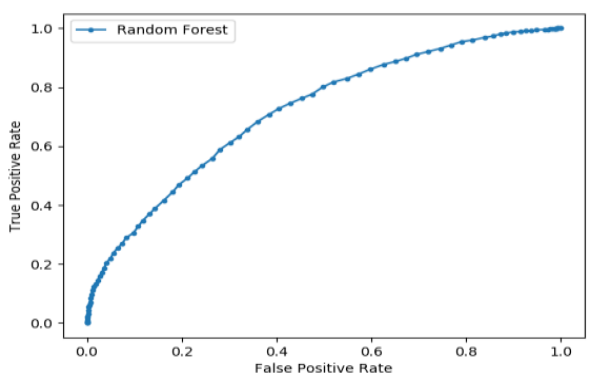

Fig. 2. (b) ROC curve of Random Forest

On the other side, Bagging with SVM(kernel='linear'), Perceptron and Passive Aggressive Classifier shows the worst performance- their accuracy scores are $48.23 \%$, $49.11 \%$ and $50.62 \%$ respectively and their F1-scores are$0.46,0.35$ and 0.51 . Moreover, SVM (with different kernels) and Decision Tree do not show good performance either. Table $\mathrm{V}$ shows the ranks classifiers of the different algorithms based on accuracy and F1-score. Table VII represents the confusion matrix for the best two classifiers i.e. Bagging with Random Forest and Bagging with Decision Tree. Figure. 3. (a) and (b) represents the ROC curve for the above two classifiers.

Table VII: Confusion Matrix to detect pre-defined level

\begin{tabular}{|c|c|c|c|c|}
\hline \multirow{2}{*}{ Classifier } & \multicolumn{4}{|c|}{ Confusion Matrix } \\
\cline { 2 - 5 } & $T P$ & $T N$ & $F P$ & $F N$ \\
\hline Bagging with Random Forest & 1285 & 1094 & 734 & 731 \\
\hline Bagging with Decision Tree & 1303 & 1072 & 756 & 713 \\
\hline
\end{tabular}

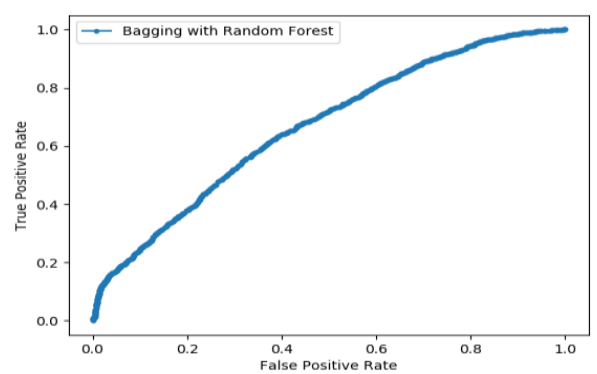

Fig. 3. (a) ROC curve of Bagging with Random Forest 


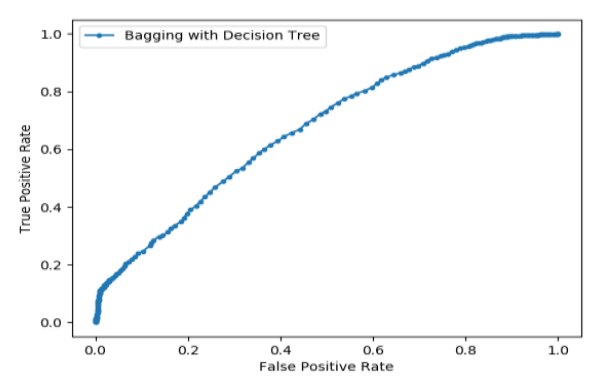

Fig. 3. (b) ROC curve of Bagging with Decision Tree

\section{CONCLUSION}

In this paper, we have used EEG data of students to predict the confusion level while watching the MOOC videos in order to improve the quality and effectiveness of online education system. We have applied 32 supervised learning algorithms with various parameters setting to detect whether a student is 'confused' or 'not confused' while watching MOOC videos. It can be seen from the results that bagging with Random Forest gives an accuracy of $61.89 \%$ for pre-defined confusion level detection and $66.6 \%$ for user-defined confusion level detection. So, we can observe that these computational approaches may use to identify the confusion level of the students. Since, the computed accuracy is less so, in future we can apply Universum based models to improve the performance.

\section{REFERENCES}

1. Allen, I. Elaine, and Jeff Seaman. Going the distance: Online education in the United States, 2011. Sloan Consortium. PO Box 1238 , Newburyport, MA 01950, 2011.

2. Thompson, Gordon. "How can correspondence-based distance education be improved?: a survey of attitudes of students who are not well disposed toward correspondence study." International Journal of E-Learning \& Distance Education/Revue internationale du e-learning et la formation à distance 5.1 (1990): 53-65.

3. Sharma, Ramesh C. "Innovative Applications of Online Pedagogy and Course Design." International Journal of Information and Communication Technology Education 15.2 (2019).

4. Sublett, Cameron. "What Do We Know About Online Coursetaking, Persistence, Transfer, and Degree Completion Among Community College Students?." Community College Journal of Research and Practice 43.12 (2019): 813-828.

5. Teplan, M. "Fundamental Of EEG Measurement\| Measurement Science Review." (2002).

6. Niemic, Christopher. "Studies of Emotion: A Theoretical and Empirical Review of Psychophysiological Studies of Emotion." (2004).

7. Chernecky, Cynthia C., and Barbara J. Berger. Laboratory Tests and Diagnostic Procedures-E-Book. Elsevier Health Sciences, 2012.

8. https://en.wikipedia.org/wiki/Electroencephalography (Accessed $4^{\text {th }}$ Nov, 2019).

9. Yeo, Mervyn VM, et al. "Can SVM be used for automatic EEG detection of drowsiness during car driving?." Safety Science 47.1 (2009): 115-124.

10.Wang, Haohan, et al. "Using EEG to Improve Massive Open Online Courses Feedback Interaction." AIED Workshops. 2013.

11. Mampusti, Ella T., et al. "Measuring academic affective states of students via brainwave signals." 2011 Third International Conference on Knowledge and Systems Engineering. IEEE, 2011.

12. Subasi, Abdulhamit, and M. Ismail Gursoy. "EEG signal classification using PCA, ICA, LDA and support vector machines." Expert systems with applications 37.12 (2010): 8659-8666.

13. Ni, Zhaoheng, et al. "Confused or not confused?: Disentangling brain activity from eeg data using bidirectional 1 stm recurrent neural networks." Proceedings of the 8th ACM International Conference on Bioinformatics, Computational Biology, and Health Informatics. ACM, 2017.
14. Edla, Damodar Reddy, et al. "Classification of EEG data for human mental state analysis using Random Forest Classifier." Procedia computer science 132 (2018): 1523-1532.

15. Mangalagowri, S. G., and P. Cyril Prasanna Raj. "EEG feature extraction and classification using feed forward backpropogation algorithm for emotion detection." 2016 International Conference on Electrical, Electronics, Communication, Computer and Optimization Techniques (ICEECCOT). IEEE, 2016.

16. Hajinoroozi, Mehdi, et al. "Feature extraction with deep belief networks for driver's cognitive states prediction from EEG data." 2015 IEEE China Summit and International Conference on Signal and Information Processing (ChinaSIP). IEEE, 2015.

17. Petrosian, A. A., et al. "Recurrent neural network-based approach for early recognition of Alzheimer's disease in EEG." Clinical Neurophysiology 112.8 (2001): 1378-1387.

18. Abdulla, Shahab, et al. "Sleep EEG signal analysis based on correlation graph similarity coupled with an ensemble extreme machine learning algorithm." Expert Systems With Applications 138 (2019): 112790.

19. Hussain, Mushtaq, et al. "Using machine learning to predict student difficulties from learning session data." Artificial Intelligence Review 52.1 (2019): 381-407.

20. Lin, Fu-Ren, Chien-Min Kao. "Mental effort detection using EEG data in E-learning contexts." Computers \&Education 122 (2018): 63-79.

21. Bacos, Catherine A. "Machine Learning and Education in the Human Age: A Review of Emerging Technologies." Science and Information Conference. Springer, Cham, 2019.

22. Raheel, Aasim, Muhammad Majid, and Syed Muhammad Anwar. "Facial Expression Recognition based on Electroencephalography." 2019 2nd International Conference on Computing, Mathematics and Engineering Technologies (iCoMET). IEEE, 2019.

23. Zhou, Yun, et al. "Confusion State Induction and EEG-based Detection in Learning." 2018 40th Annual International Conference of the IEEE Engineering in Medicine and Biology Society (EMBC). IEEE, 2018.

24. https://www.kaggle.com/wanghaohan/confused-eeg

\section{AUTHORS PROFILE}

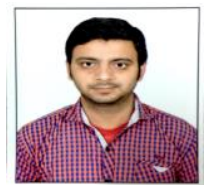

Bikram Kumar is pursuing M.Tech in Computer Science and Engineering of National Institute of Technology, Arunachal Pradesh. He has completed B.Sc from Gour Mahavidyalaya and M.Sc from University of Gour Banga in Computer Science. His area of interests include Machine Learning, Data Science and Data Mining.

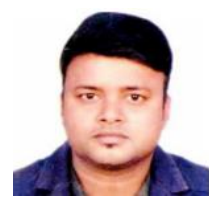

Dr. Deepak Gupta is currently working as Assistant Professor in Computer Scinece and Engineering of National Institute of Technology, Arunachal Pradesh. He has completed MCA from Jawaharlal Neheru University. He has also completed M.Tech and Ph.D in Computer Science and Engineering from Jawaharla Neheru University. His research fields include Machine Learing, Support Vector Machine, Extreme Learning Machine for Classification and Regression Problems. In 2017, he received the SERB-Early Career Research Award in Engineering Sciences.

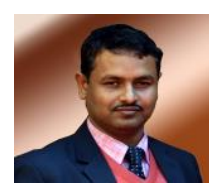

Dr. Rajat Subhra Goswami is currently working as Assistant Professor in Computer Science and Engineering of National Institute of Technology, Arunachal Pradesh. He has completed B.Tech in Information Technology from West Bengal University of Technology, M.Tech in Multimedia Development from Jadavpur University and Ph.D in Computer Science and Engineering from National Institute of Technology, Arunachal Pradesh. His research fields include Information Security, Cryptography, Image Processing, Big Data, Network Traffic Classification. 